\title{
COVID-19 Vaccination Challenges in Developing Countries
}

\author{
Wajahat Hussain ${ }^{1}$
}

1. Department of Community Medicine, Quaid-e-Azam Medical College, Bahawalpur, Pakistan

\section{*Correspondence:} wajahatbukhari@gmail.c om

Keywords: COVID-19, social media, pandemic

doi:

10.37978/tijfs.v5i1.356

How to cite this:

Bukhari, W. COVID-19

Vaccination Challenges in Developing Countries. Int J Front Sci, 5(1). 1-2.

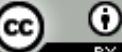

This article is open access under terms of Creative Commons Attribution License 4.0. which permits unrestricted use, distribution and reproduction in any medium provided the original work is cited properly.
The pandemic of coronavirus disease 2019 (COVID-19) is challenge of the century for humanity. Pre-pandemic normalcy is assumed to never return until a safe and effective vaccine becomes available and a global vaccination campaign is successfully introduced. To tackle the pandemic of Covid-19 safe and effective vaccines has been developed and pharmaceutical companies Pfizer/BioNTech, Moderna and AstraZeneca started to manufacture the vaccine and make it available in the market. Globally all the countries are in race to secure vaccine access for their populations but it is challenge for developing countries to make vaccine available for the population especially the poor and vulnerable groups. Pakistan is developing country which is facing the double burden of the communicable diseases like malaria, HIV/AIDS, tuberculosis and non-communicable diseases along with financial issues faced by the health system. ${ }^{1,2}$

For stopping the transmission of COVID-19 disease it is the need of the hour that along with all the precautionary measures like social distancing and hand washing the global access to the vaccines must be ensured. Key challenges faced by the developing countries including Pakistan for universal access of vaccine to their populations may include limited vaccine availability due to manufacturing capacity, affordability and vaccine storage or cold chain problems. Poor transport infrastructure and a lack of adequate refrigeration facilities may also hamper the distribution of vaccines. Access for internally displaced people and refugees is another issue. The major barrier for access of vaccine may be the cost in developing nations. The estimated price of Oxford vaccine at maximum of $\$ 3$ per dose for Lower and Middle Income Countries (LMICs) and \$4-\$5 elsewhere. Pfizer company has been agreed a cost with the US Administration of $\$ 19.50$ a dose. It may become a benchmark for developing nations. Moderna is charging \$32 to \$37 a dose for smaller deals and less for larger orders. . $^{3,4}$

The risk of severe morbidity and mortality due to COVID-19 is high among elderly and people with comorbidities like diabetes mellitus, malignancies, Chorionic liver \& kidney diseases. The rate of infection is also high among health care providers as they are directly in contact with people suffering from COVID-19 and they can transmit the infection to large number of patients and their attendants admitted or visiting the hospital. For COVID-19 those at higher risk of severe infection (including elderly, individuals with medical conditions that predispose them to severe disease and healthcare providers) should be prioritized for access to vaccine.

Vaccines can lose their effectiveness if they are not kept within a narrow temperature range. Most are heat sensitive: warm temperatures kill the live-attenuated viruses or bacteria within, reducing potency. Many are also freezesensitive: extreme cold can corrupt adjuvants added to vaccines to help them work and ultimately reducing their effectiveness. The COVID-19 vaccine made by one of the front-runners in vaccine race (Pfizer) needs to be kept at minus 70 degrees Celsius, which is colder than winter in Antarctica. Moderna vaccine also needs to be frozen too, but only at minus 20 Celsius, more like a regular freezer. ${ }^{5,6}$ Since there will be limited vaccine doses will be available in Pakistan initially the immunization managers across the country will need to have plans to distribute all vaccine doses that are available at the recommended temperature to maintain their potency. It will be a big challenge presented by the Pfizer vaccine, which requires these ultra-cold conditions and if not addressed timely it will result in loss of vaccine efficacy and ultimately all the cost and efforts devoted for prevention of disease through vaccination will be useless.

\section{References}

1. Jeyanathan M, Afkhami S, Smaill F, Miller MS, Lichty BD, Xing Z. Immunological considerations for COVID-19 vaccine strategies. Nature Reviews Immunology. 2020 Oct;20(10):615-32.

2. Khan YH, Mallhi TH, Alotaibi NH, Alzarea AI, Alanazi AS, Tanveer N, Hashmi FK. Threat of COVID-19 vaccine hesitancy in Pakistan: the need for measures to neutralize misleading narratives. The American journal of tropical medicine and hygiene. 2020 Aug 5;103(2):603-4.

3. Phelan AL, Eccleston-Turner M, Rourke M, Maleche A, Wang C. Legal agreements: barriers and enablers to global equitable COVID-19 vaccine access. The Lancet. 2020 Sep 19;396(10254):800-2.

4. Persad G, Peek ME, Emanuel EJ. Fairly prioritizing groups for access to COVID-19 vaccines. Jama. 2020 Oct 27;324(16):1601-2.

5. Mills MC, Salisbury D. The challenges of distributing COVID-19 vaccinations. EClinicalMedicine. 2020 Dec 8.

6. National Academies of Sciences, Engineering, and Medicine. Framework for equitable allocation of COVID-19 vaccine. National Academies Press; 2020 Nov 30. 
